\title{
Efficacy and safety of electronic brachytherapy for superficial and nodular basal cell carcinoma
}

\author{
Rosa Ballester-Sánchez, MD!, Olga Pons-Llanas, MD², Cristian Candela-Juan, PhD³, Francisco Javier Celada-Alvarez, MD²

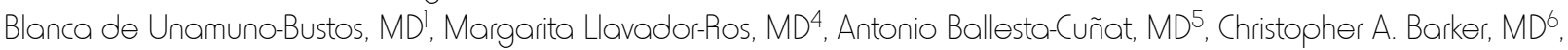 \\ Alejandro Tormo-Mico, MD², Rafael Botella-Estrada, MDl, Jose Perez-Calatayud, PhD ${ }^{3}$
}

\begin{abstract}
'Dermatology Department, La Fe University and Polytechnic Hospital, Valencia, Spain, ${ }^{2}$ Radiotherapy Department, La Fe University and Polytechnic Hospital, Valencia, Spain, ${ }^{3}$ Radiation Physics Department, La Fe University and Polytechnic Hospital, Valencia, Spain, ${ }^{4}$ Pathology Department, La Fe University and Polytechnic Hospital, Valencia, Spain, ${ }^{5}$ Radiology Department, La Fe University and Polytechnic Hospital, Valencia, Spain, 'Department of Radiation Oncology, Memorial Sloan Kettering Cancer Center, New York, USA
\end{abstract}

\begin{abstract}
Purpose: Surface electronic brachytherapy (EBT) is an alternative radiotherapy solution to external beam electron radiotherapy and high-dose-rate radionuclide-based brachytherapy. In fact, it is also an alternative solution to surgery for a subgroup of patients. The objective of this work is to confirm the clinical efficacy, toxicity and cosmesis of a new EBT system, namely Esteya ${ }^{\circledR}$ in the treatment of nodular and superficial basal cell carcinoma (BCC).

Material and methods: This is a prospective single-center, non-randomized pilot study to assess the efficacy and safety of EBT in nodular and superficial BCC using the Esteya ${ }^{\circledR}$ system. The study was conducted from June 2014 to February 2015. The follow up time was 6 months for all cases.

Results: Twenty patients with 23 lesions were included. A complete response was documented in all lesions (100\%). A low level of toxicity was observed after the $4^{\text {th }}$ fraction in all cases. Erythema was the most frequent adverse event. Cosmesis was excellent, with more than $60 \%$ of cases without skin alteration and with subtle changes in the rest.

Conclusions: Electronic brachytherapy with Esteya ${ }^{\circledR}$ appears to be an effective, simple, safe, and comfortable treatment for nodular and superficial BCC associated with excellent cosmesis. It could be a good choice for elderly patients, patients with contraindications for surgery (due to comorbidities or anticoagulant drugs) or patients where surgery would result in a more disfiguring outcome. A longer follow-up and more studies are needed to confirm these prelim-
\end{abstract} inary results.

Key words: basal cell carcinoma, electronic brachytherapy, skin brachytherapy, skin cancer.

\section{Purpose}

Basal cell carcinoma (BCC) is the most common skin cancer, accounting for approximately $75 \%$ of all skin cancers. It is also the most common subtype of non-melanoma skin cancer (NMSC), representing $80-90 \%$ of the total [1]. Its incidence is increasing due to an aging general population as well as increased exposure to ultraviolet radiation, resulting in a great care burden and therefore a high healthcare spend [2]

The therapeutic management is based on the application of invasive therapies (conventional surgery or Mohs surgery) or noninvasive therapies (cryotherapy, curettage and electrodessication, topical imiquimod, photodynamic therapy, and radiation). These modalities are asso- ciated with different response rates, complications, and cosmetic results. The choice of treatment mainly depends on the histologic subtype, location, and patient comorbidities. Surgery is the first choice because of the often simple procedure and high efficacy [3]. However, despite the high incidence of BCC, very few randomized studies have been undertaken to compare treatment modalities. In a meta-analysis of the Cochrane Library, published in 2007, a total of 27 studies were collected, and the authors concluded that surgery and radiation therapy (RT) are the treatments with the lowest rates of recurrence [3].

There are several RT techniques for treating BCC. In external beam electron RT an electron beam produced with a linear accelerator is emitted towards the patient surface. This usually requires a specific dosimetry and bolus,
Address for correspondence: Rosa Ballester-Sánchez, MD, Dermatology Department, La Fe University and Polytechnic Hospital, Valencia, Spain, Bulevar sur s/n, 46026 Valencia, Spain, phone: +34 961244186, e-mail: roseta999@hotmail.com
Received: 13.03 .2015

Accepted: 21.05 .2015

Published: 25.06.2015 
i.e., a water equivalent material placed at the patient surface with two purposes: 1) to reduce the therapeutic depth and also the total electron range, and 2) to raise the full dose on the skin because the small skin underdose in electron low energies. This makes the use of electrons more difficult in clinical practice than other RT options. An alternative option is radionuclide based high-doserate (HDR) brachytherapy (BT). It uses an ${ }^{192}$ Ir HDR radioactive source placed near the lesion, which emits radiation. It can be performed with moulds and flaps or with dedicated skin applicators. Brachytherapy with skin applicators is an efficient solution for superficial RT of small lesions, providing a higher shielding to the surrounding healthy tissues when compared to moulds and flaps [4].

High-dose-rate surface electronic brachytherapy (EBT) is an alternative solution to external beam electron RT and HDR radionuclide-based BT. This EBT solution uses $X$-ray generators with specific applicators that collimate the beam and that are placed in contact with the patient surface. Currently, there are three EBT systems for treatment of surface lesions: the $50 \mathrm{kVp}$ Xoft Axxent ${ }^{\circledR}(\mathrm{iCad}$, San Jose, CA, USA) with 1, 2, 3.5, and $5 \mathrm{~cm}$ diameter applicators [5,6], the $50 \mathrm{kVp}$ Zeiss INTRABEAM ${ }^{\circledR}$ (Carl Zeiss Surgical Gmbh, Oberkochen, Germany), with $1 \mathrm{~cm}$ to $6 \mathrm{~cm}$ diameter applicators $[7,8]$, and the most recent $69.5 \mathrm{kVp}$ Esteya $^{\circledR}$ (Elekta Brachytherapy, Veenendaal, The Netherlands), provided with $1 \mathrm{~cm}$ to $3 \mathrm{~cm}$ diameter applicators [9].

The main objective of this work is to confirm the clinical efficacy of EBT with the Esteya ${ }^{\circledR}$ system in the treatment of nodular and superficial BCC. The specific objectives are: 1) to determine the toxicity by CTCAE scale and 2) to evaluate the cosmetic results by RTOG-EORTC scale.

This is the first phase of a study that will be completed after 2 years follow-up.

\section{Material and methods}

\section{Description of the X-ray source and surface applicators}

The EBT system used in this study was Esteya ${ }^{\circledR}$, with a $69.5 \mathrm{kV}$ X-ray source and an aluminum flattening filter that produces a homogenous beam at the patient's skin surface $[10,11]$. Esteya ${ }^{\circledR}$ comes with a set of applicators that are able to generate circular radiation fields of different diameters: $10 \mathrm{~mm}, 15 \mathrm{~mm}, 20 \mathrm{~mm}, 25 \mathrm{~mm}$, and $30 \mathrm{~mm}$. Each surface applicator has a plastic cap designed to maintain constant source to surface distance (SSD). With the applicator in place, the nominal SSD is $6 \mathrm{~cm}$. The source is connected, controlled, and powered by a controller, which supplies a high voltage and filament current. The default current is $1.6 \mathrm{~mA}$, which is automatically set to $1.0 \mathrm{~mA}$ for treatment fractions smaller than $4 \mathrm{~Gy}$, and to $0.5 \mathrm{~mA}$ for prescription doses below $2 \mathrm{~Gy}$. The fraction duration is therefore kept relatively constant, between 2 and 3 minutes for commonly used fraction sizes, and is independent of the prescribed dose. The patient's treatment plan is controlled through a specific software that only requires information about prescription dose, number of fractions, prescription depth, and applicator size. With these data, the system automatically computes the treatment time.

\section{Study design}

This is a prospective, single-center, non-randomized, pilot study to assess the efficacy and safety of EBT in superficial and nodular BCC treatment using Esteya ${ }^{\circledR}$ surface applicators. Only BCC was included in this study due to its high frequency and its biological behavior with local invasion and low metastatic capacity. The study was conducted from June 2014 to February 2015. It was approved by the ethics committee of clinical research of La Fe Hospital. All patients or legal guardians signed a written informed consent.

\section{Methodology}

Stage T1 or T2 (according to AJCC 2010) [12] superficial or nodular BCCs, confirmed by punch biopsy, were included. Due to the applicator design, the maximum diameter of the lesion should have been $20 \mathrm{~mm}$. The maximum depth of lesion included in this study was $4 \mathrm{~mm}$, measured by biopsy and ultrasonography. Lesions can be treated at any skin surface, except irregular or not flat surfaces. The step by step procedure is described in a previous work [9].

Patient demographics and lesion characteristics were recorded. Fitzpatrick scale was used to classify patients by phototype: 1) always burns, never tans; 2) usually burns, tans minimally; 3) sometimes mild burn, tans uniformly; 4) rarely burns, always tans well; 5) very rarely burns, tans very easily; 6) never burns, tans very easily.

\section{Treatment}

The gross tumor volume (GTV) was assessed clinically with the aid of a dermoscope [13]. A radial margin of $5 \mathrm{~mm}$ was added to establish the clinical target volume (CTV) and the minimum applicator that covered the whole CTV was selected $(10,15,20,25$ or $30 \mathrm{~mm})$. A specific applicator template named templates La Fe [9] was used in order to delineate a mark on the skin to fit the external diameter of the selected applicator (Fig. 1). Tumor depth was assessed by high frequency ultrasonography (HFUS) and histopathology. A minimum depth of $3 \mathrm{~mm}$ by convention was used for lesions having a depth of $3 \mathrm{~mm}$ or less, and for deeper lesions the specific lesion depth was used for prescription, with a maximum of $5 \mathrm{~mm}$ [14]. After attaching the selected applicator to the machine, it was centered on the lesion using the mark made, and positioned in full contact with the lesion. The Esteya ${ }^{\circledR}$ arm has several degrees of freedom in order to place the applicator (Fig. 2).

The prescription dose was $42 \mathrm{~Gy}$, divided in 6 fractions of $7 \mathrm{~Gy}$, delivered twice weekly with at least 48 hours between each fraction, according to the experience of Tormo et al. with the Valencia applicator [15]. Treatment times were calculated automatically by the application console, once the applicator size, dose, and depth were introduced and verified by the operator [11]. Treatment duration was less than 3 minutes in all cases. 

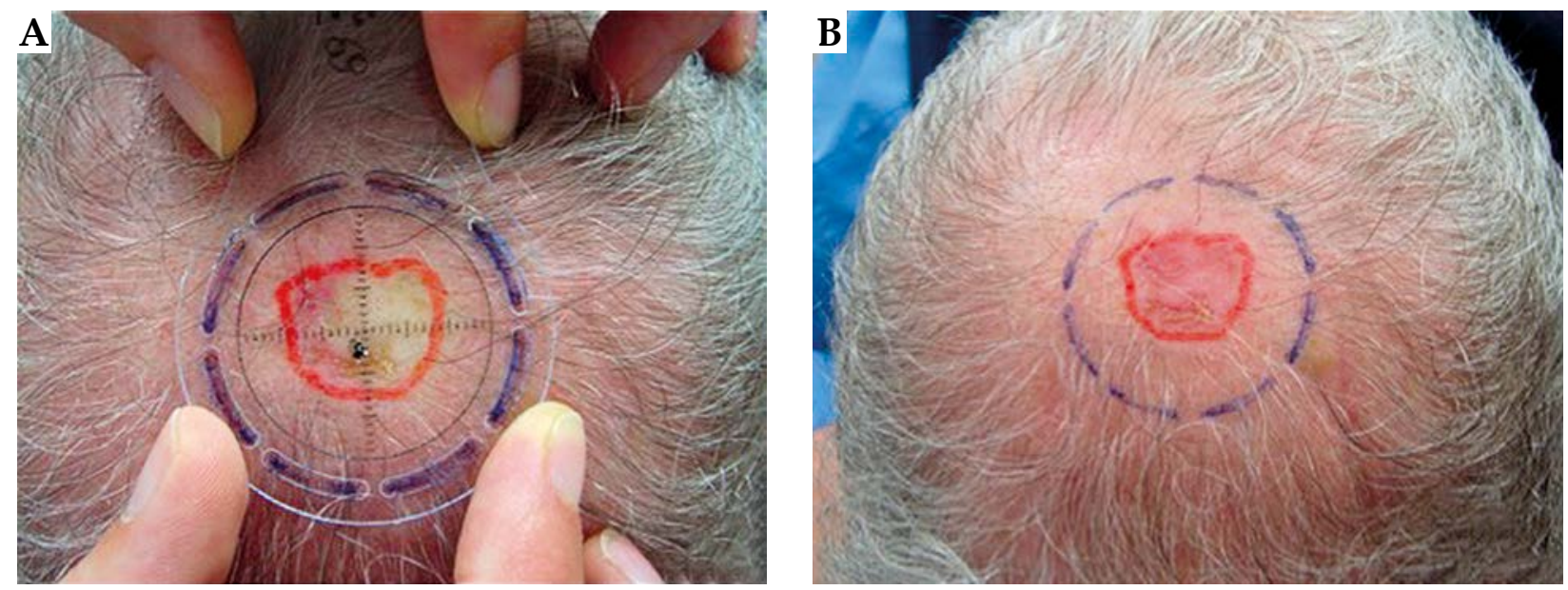

Fig. 1. A) Tumor marking with template La Fe. B) Marks delimiting tumor margin (in red) and the external mark (in blue) to fit the applicator in the center

\section{Follow up and evaluation}

After treatment, patients were instructed to avoid sun exposure and to apply a sunscreen if sun exposure was unavoidable. If lesions became ulcerated during treatment, a petrolatum ointment was applied as needed. Patients were seen for follow up at 2 and 6 weeks after treatment in order to assess cutaneous toxicity, and at 3 and 6 months to evaluate efficacy and cosmesis. Clinical and dermoscopic photographs were taken at each visit. Additional follow up visits are planned at one and two years after treatment.

CTCAE v4.0 (Common Terminology Criteria for Adverse Events) toxicity scales were used to assess acute toxicity and RTOG-EORTC scales related to brachytherapy were used to assess late toxicity related to cosmesis (Tables 1 and 2).

\section{Statistics}

Data are reported as mean \pm standard deviation. Categorical variables are presented as proportions. Differences between groups were determined using the Fisher exact test (due to the presence of a percent $<5 \%$ in one group). Finally, stepwise multivariate logistic regression model was used to identify all independent predictors of toxicity. Statistical analysis was performed with SPSS Statistics $18^{\circledR}$ (SPSS Inc, Chicago, USA) program. We considered $p$ values $<0.05$ significant.

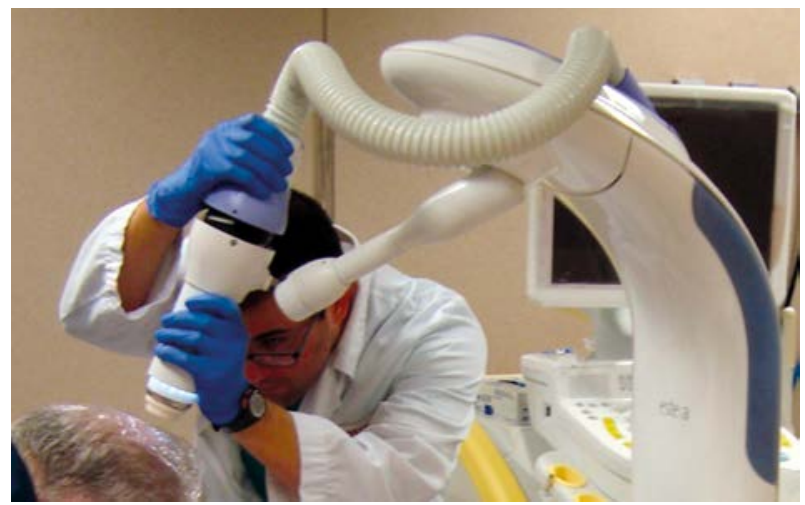

Fig. 2. The Esteya ${ }^{\circledR}$ arm has several degrees of freedom in order to place the applicator

\section{Results}

\section{Patient demographics}

Twenty patients with 23 lesions were included in this study. Baseline characteristics of patients and lesions are summarized in Table 3 . The mean age was 79 years (range 64-91), with a male predominance. All patients had one or more comorbidities. More than a quarter of patients had heart disease and/or were undergoing treatment with antiplatelets or anticoagulants. All patient were Caucasian and most of them were phototype III.

Table 1. CTCAE (Common Terminology Criteria for Adverse Events) related to skin

\begin{tabular}{lccccc} 
Toxicity & G1 & G2 & G3 & G4 & G5 \\
\hline Atrophy & Mild & Marked & & & \\
\hline Alopecia & $<50 \%$ & $250 \%$ & & & \\
\hline Pigmentation change & Mild or localized & Marked or generalized & & & \\
\hline Skin ulceration & $<1 \mathrm{~cm}$ & $1-2 \mathrm{~cm}$ & $>2 \mathrm{~cm}$ & Deep structures involvement & Death \\
\hline Erythema & Mild & Moderate & Severe & Necrosis & Death
\end{tabular}

Original source: adapted from NCI CTCAE V4.0. 
Table 2. Cutaneous brachytherapy related RTOG EORTC (Radiation Therapy Oncology Group and the European Organization for Research and Treatment of Cancer)

\begin{tabular}{lccc} 
G1 & G2 & G3 & G4 \\
\hline Slight atrophy & Patchy atrophy & Marked atrophy & Ulceration \\
Pigmentation & Moderate & Gross & \\
change & telangiectasias & telangiectasias & \\
Some hair loss & Total hair loss & &
\end{tabular}

Original source: adapted from RTOG/EORTC late radiation morbidity.

Table 3. Baseline patients and lesion characteristics

\begin{tabular}{lc} 
Women, $n$ (\%) & $8(40)$ \\
\hline Age (years) & $79 \pm 1.86$ \\
\hline Phototype, $n$ (\%) & $9(45)$ \\
\hline II & $11(55)$ \\
\hline III & $2(10)$ \\
\hline Comorbidities, $n$ (\%) & $5(25)$ \\
\hline Peacemaker & $3(15)$ \\
\hline Cardiopathy & $6(30)$ \\
\hline Dementia & $17(85)$ \\
\hline Antiplatelet/anticoagulant, $n(\%)$ & $3(15)$ \\
\hline Number of BCC, $n$ (\%) & \\
\hline 1 & $16(70)$ \\
\hline 2 & $4(17)$ \\
\hline Lesion locations, $n(\%)$ & $3(13)$ \\
\hline Head and neck & \\
\hline Extremities & \\
\hline Trunk & $11(48)$ \\
\hline Lesion diameter (mm): applicator & $10(44)$ \\
\hline size (mm), $n(\%)$ & $2(8)$ \\
\hline$\leq 10: 20$ & $2.62(2.56-2.83)$ \\
\hline $11-15: 25$ & \\
\hline $16-20: 30$ & \\
\hline Dose depth (mm), $n(\%)$ & \\
\hline 3 & \\
\hline 4 & \\
\hline Treatment time (minutes) & \\
\hline
\end{tabular}

\section{Lesion characteristics}

The most common location was head and neck $(70 \%)$. The maximum diameter of lesions ranged from 8 to $20 \mathrm{~mm}$ (average $11.9 \mathrm{~mm}$ ). Most of the lesions had a diameter between 8 and $15 \mathrm{~mm}$. The size of applicators most frequently used were $20 \mathrm{~mm}(48 \%)$ and $25 \mathrm{~mm}(44 \%)$. Nodular type BCC was predominant (61\%), 30\% of cases were pigmented and only $4 \%$ were ulcerated. All tumors were stage T1 according to AJCC 2010. Mean tumor thickness was $1.57 \mathrm{~mm}$ measured by histopathology (range 0.252.9 ) and $1.90 \mathrm{~mm}$ measured by ultrasonography (range 0.5-3.7). The depth prescription was $3 \mathrm{~mm}$ in $87 \%$ of lesions and $4 \mathrm{~mm}$ in the other cases, as justified in Ballester et al. [13]. The treatment time per fraction ranged from $2.56 \mathrm{~min}\left(2^{\prime} 33^{\prime \prime}\right)$ to $2.83 \mathrm{~min}\left(2^{\prime} 50^{\prime \prime}\right)$, with a mean value of $2.62 \min \left(2^{\prime} 37^{\prime \prime}\right)$.

\section{Efficacy}

A complete response was documented for all lesions $(100 \%)$. We took clinical and dermoscopic photographs at 2 weeks, 6 weeks, 3 months, and 6 months after treatment (see Figs. 3 and 4). A punch biopsy was planned at 6 months if there was suspicion of residual disease, but this has not occurred. Minimum follow-up was 6 months.

\section{Toxicity}

Toxicity observed during treatment started after the $4^{\text {th }}$ fraction and was most severe between 2 and 6 weeks after treatment. Toxicity was assessed using the CTCAE (Common Terminology Criteria for Adverse Events) scale (Table 1). G1 or G2 toxicity was observed in all cases. Erythema was the most frequent adverse event (100\%). Ulceration and crusting appeared most frequently in nodular cases, in trunk, and extremities. A significantly higher incidence of ulceration in trunk and extremities cases compared with head and neck cases was found (100\% vs. 44.4 $\pm 0.2 \%, p=0.003)$. In the regression model, we introduced the following variables: age, antithrombotic treatment, Breslow ( $\mathrm{mm})$, depth prescription, tumor diameter, and tumor location. The only independent predictor of toxicity was a trunk and extremities tumor location (OR $=9.77, \mathrm{CI} 95 \%$ : $5.02-15.21, p=0.003$ ). We did not find a statistically significant relationship between toxicity and other parameters.

No other adverse events were reported.

\section{Cosmetic results}

Cosmesis is related to long-term toxicity. It was measured with the brachytherapy related RTOG-EORTC scale (Radiation Therapy Oncology Group and the European Organization for Research and Treatment of Cancer) (Table 2). The potential adverse events with RT are: radiodermitis, itching, pain, ulceration, alopecia, hyperpigmentation, hypopigmentation, telangiectasia, skin atrophy, and induration. We observed only pigmentation changes (central hypopigmentation with peripheral hyperpigmentation) and alopecia. Sixty-one percent of the lesions showed no skin alteration (G0). The remainder showed subtle changes (G1). When comparing the cosmetic events in head and neck cases with trunk and extremities cases, we found a significantly lower percentage of cosmetic events in the head and neck cases (37.5 $\pm 0.12 \%$ vs. $100 \%, p=0.007$ ). Finally, incidence of pigmentation changes in each phototype group was studied. A higher incidence of pigmentation change was found in the phototype III group $(42.9 \pm 0.13 \%$ vs. $11.1 \pm 0.1 \%$ in the phototype II group, $p=0.062$ ).

\section{Discussion}

This is the first study performed with the new electronic brachytherapy system Esteya ${ }^{\circledR}$, applying the fractionation protocol successfully used with the radionuclide 



Fig. 3. A) Superficial basal cell carcinoma located on the scalp prior to treatment. B) Two weeks after last electronic brachytherapy (EBT) fraction. C) Three months after last EBT fraction. D) Six months after last EBT fraction

based Valencia applicator [15]. The potential radiobiological correction due to the lower energy has not been taken into account and the same physical dose has been applied.

Several studies have evaluated the effectiveness of RT in the treatment of BCC. Most are retrospective and use highly variable radiation doses. Cho et al. [16] recently reviewed all published studies on the use of RT in BCC and identified 11 studies with response rates of $79.2 \%$ to $100 \%$, and cure rates at 5 years of 79.2 to $95.8 \%$ (evaluated in 4 studies). Currently, there are only two randomized studies evaluating the efficacy of RT in BCC. Hall et al. [17] compared the efficacy of RT and cryotherapy in a series of 93 patients and identified a higher recurrence rate in the group treated with cryotherapy (39\%) compared to the radiotherapy group (4\%), without identifying significant differences in cosmetic results. Avril et al. [18] treated 347 BCCs located on the face with either radiotherapy or surgery, and followed patients for 41 months. Only one patient in the surgery group presented with a tumor recurrence compared with 11 patients in the radiotherapy group. Based on these results, they concluded that surgery should be a first line treatment.

It is important to note that patients referred to RT often have unresectable tumors or contraindications for surgery due to advanced age or comorbidities. This may induce a bias in interpreting the results and comparing them with other therapeutic procedures. Some authors have suggested that RT could be an effective therapeutic alternative for patients with limited life expectancy, where one can get good rates of tumor control and a good cosmetic result. Nguyen et al. [19] conducted a retrospective study that included 15 patients with a mean age of 81 years, who underwent treatment with RT at doses between $45 \mathrm{~Gy}$ and $72.5 \mathrm{~Gy}$. After a mean follow-up of 34 months, no loco-regional recurrences were observed, with a complete response being reached in all patients and a very good cosmetic result.

Electronic brachytherapy is a type of contact radiotherapy, developed in recent years, that uses low energy $\mathrm{X}$-ray sources in order to direct the radiation dose to the size and shape of the tumor, while preserving the surrounding healthy tissues. Only one study has been reported to date [20], describing the results obtained by applying a total dose of $40 \mathrm{~Gy}$ (8 fractions of $5 \mathrm{~Gy}$ ) in 171 NMSCs, delivered with the EBT source of $50 \mathrm{kV}$. In this series, complete responses were obtained in $100 \%$ of the treated lesions, with no recurrences after a year of monitoring and good cosmetic results. It should be noted that one year follow up data was only available for a subset (42) of the patients treated. 

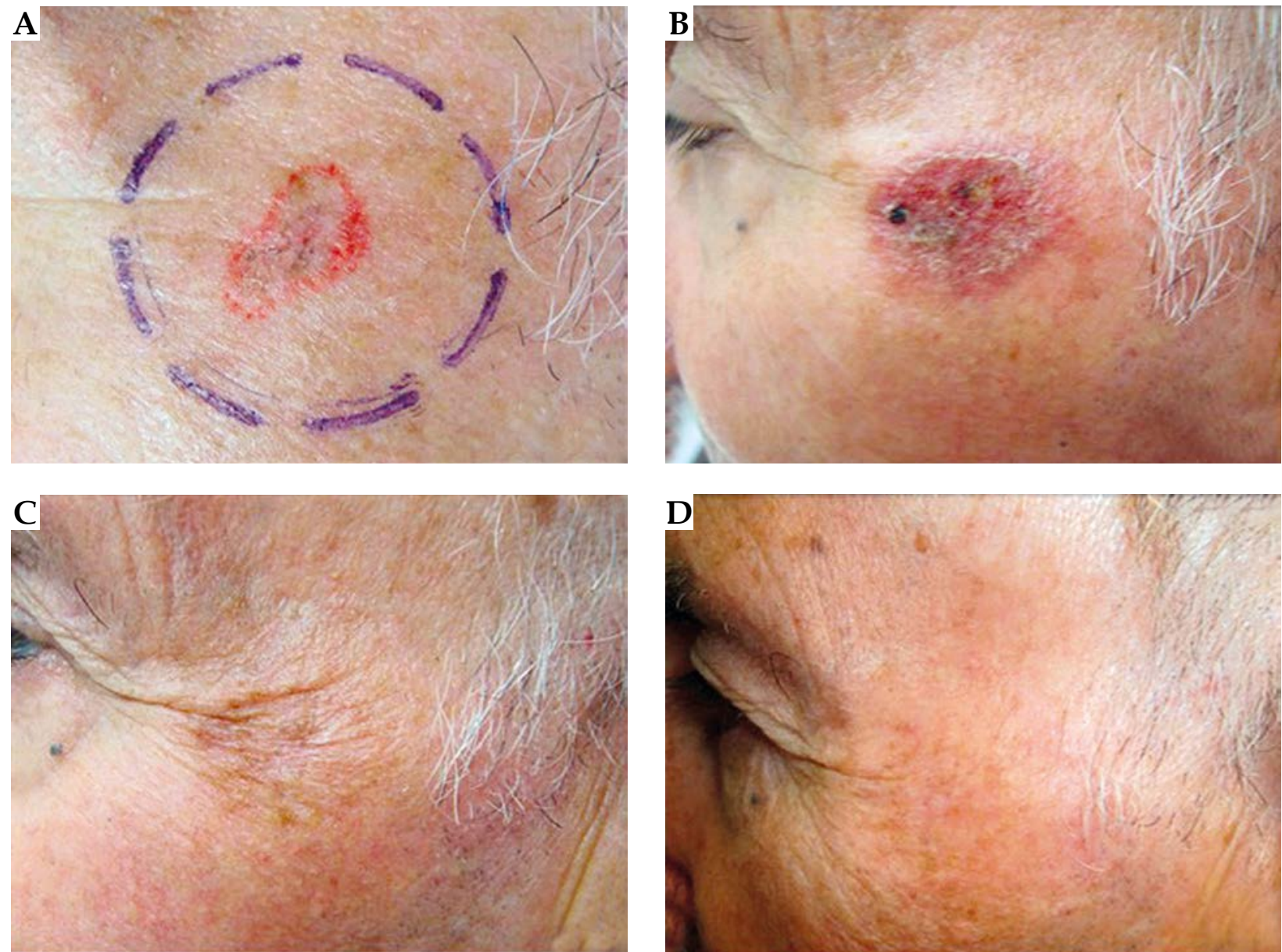

Fig. 4. A) Superficial basal cell carcinoma located on the cheek prior to treatment. B) Two weeks after last EBT fraction. C) Three months after last EBT fraction. D) Six months after last EBT fraction

Our study shows that electronic brachytherapy for superficial and nodular BCCs is effective, safe, and comfortable for the patients and is associated with very good cosmesis. We found a significantly higher incidence of ulceration in trunk and extremities cases, compared with head and neck cases. When comparing the cosmetic events in head and neck cases with trunk and extremities cases, we found a significantly lower percentage of cosmetic events in the head and neck cases. We also found a relation between pigmentation changes and phototype, with a higher incidence of pigmentation changes in higher skin phototypes.

Tumor local control with radiotherapy depends heavily on a correct definition of margins and tumor volume in the treatment planning process. It is important to note that very narrow margins can result in inadequate coverage of the tumor to be treated, whilst margins, which are too large may increase the amount of irradiated healthy tissue. For a correct definition of margins it is necessary to define the GTV (gross tumor volume), CTV (clinical target volume), and PTV (planning target volume). Most studies determine the GTV by a delimitation of the clinically visible tumor and then adding a $5-10 \mathrm{~mm}$ margin to delimit the CTV, which represents microscopic tumor extension [21]. Dermoscopy is a simple, quick, and non-invasive technique that has been shown to increase the diagnostic accuracy in skin tumors. Several studies have demonstrated the usefulness of dermoscopy in the pre-surgical division of $\mathrm{BCC}$, achieving a higher rate of complete tumor resections, and better sparing of peritumoral healthy tissue $[22,23]$. In this way and in our opinion, the use of dermoscopy is of great value for the delimitation or BCC prior to radiotherapy [13].

Another aspect to consider before starting RT is tumor depth determination since this determines the prescription dose. The gold standard for determining the depth is the incisional biopsy, however, it has the disadvantage that it only allows analysis of a small portion of tumor tissue. The recent development of high frequency ultrasound for skin (HFUS) allows visualization of the different skin layers. Several published studies compare the findings obtained by ultrasonography and histology, and identify high rates of concordance between both techniques [1]. By contrast, other studies have found lower rates of concordance $[14,24]$. Therefore, even though the ultrasound does not replace histology, it could represent a useful tool for estimating tumor size, margin delineation before treatment, establishment of a therapeutic plan, identification of high-risk lesions, and follow up [25].

We do realize that our study has limitations: firstly, only a limited number of patients have been included. 
Secondly, as it was a phase II study to assess safety and efficacy, we did not compare the technique with other treatments. Finally, at present, the short follow up is insufficient to assess efficacy. However, our results are in line with previous publications on efficacy and safety of radiation therapy in the treatment of non-melanoma skin cancer patients. We will continue to follow the patients for at least 2 years and monitor for long term cosmesis and recurrences. Although this is the first part of a 2-year follow up study, we believe that these early results could be of interest to the new users in the implementation of this EBT system.

Electronic brachytherapy has several advantages compared with surgery and with other RT modalities. Electronic brachytherapy is comfortable for the patients because it is quick (less than 3 minutes) and painless. Fractionation twice weekly during three weeks allows the patient to be treated in a short time, in a comfortable schedule for elderly patients. The use of an electronic source avoids the storage requirements associated with radioisotopes and makes the treatment safer for patients and practitioners. Compared to the Valencia applicator, Esteya ${ }^{\circledR}$ provides better penumbra, very small leakage, less shielding requirements, and significantly shorter treatment time [10].

Esteya ${ }^{\circledR}$ is a good choice for elderly patients, patients with contraindications for surgery due to comorbidities or anticoagulant drugs or patients where surgery would result in a more disfiguring outcome.

\section{Conclusions}

For nodular and superficial BCC, EBT with Esteya ${ }^{\circledR}$ is an effective, simple, safe, and comfortable treatment associated with excellent cosmesis. It is a good treatment alternative for elderly patients, patients who do not want surgery, patients with multiple cancerous lesions, patients with contraindications for surgery (due to comorbidities or anticoagulant drugs) or patients where surgery would result in a more disfiguring outcome. A longer follow-up and more studies are needed to confirm these preliminary results. In this initial phase, it has been shown that a well proven hypofractionation regimen with the Valencia applicators can be applied successfully with the Esteya ${ }^{\circledR}$ EBT.

\section{Acknowledgements}

This study was supported by (Nucletron - an Elekta Company, Veenendaal, The Netherlands) and in part by Generalitat Valenciana (Project PROMETEOII/2013/010) and by Spanish Government under Project No. FIS201342156.

\section{Disclosure}

Authors report no conflict of interest.

\section{References}

1. Bobadilla F, Wortsman X, Muñoz C et al. Pre-surgical high resolution ultrasound of facial basal cell carcinoma: Correlation with histology. Cancer Imaging 2008; 8: 163-172.
2. Aguilar Bernier M, Rivas Ruiz F et al. Comparative epidemiological study of non-melanoma skin cancer between Spanish and north and central European residents on the Costa del Sol. J Eur Acad Dermatol Venerol 2012; 26: 41-47.

3. Bath-Hextall FJ, Perkins W, Bong J et al. Interventions for basal cell carcinoma of the skin. Cochrane Database Syst Rev 2007; 24: CD003412.

4. Marín A, Vargas Díez E, Cerezo L. Radioterapia en dermatología. Actas Dermosifiliogr 2009; 100: 166-181.

5. Rivard MJ, Davis SD, De Werd LA et al. Calculated and measured brachytherapy dosimetry parameters in water for the Xoft Axxent X-Ray Source: An electronic brachytherapy source. Med Phys 2006; 33: 4020-4032.

6. Fulkerson RK, Micka JA, Werd LA. Dosimetric characterization and output verification for conical brachytherapy surface applicators. Part I. Electronic brachytherapy source. Med Phys 2014; 41: 022103.

7. Lam SCP, Xu Y, Ingram G et al. Dosimetric characteristics of INTRABEAM ${ }^{\circledR}$ flat and surface applicators. Transl Cancer Res 2014; 3: 106-111.

8. Schneider F, Clausen S, Thölking T et al. A novel approach for superficial intraoperative radiotherapy (IORT) using a $50 \mathrm{kV}$ x-ray source: a technical and case report. J Appl Clin Med Phys 2014; 5: 4502.

9. Pons-Llanas O, Ballester-Sánchez R, Celada-Álvarez FJ et al. Clinical implementation of a new electronic brachytherapy system for skin brachytherapy. J Contemp Brachytherapy 2014; 6: 417-423.

10. Garcia-Martinez T, Jan-Pieter C, Perez-Calatayud J et al. Dosimetric characteristics of a new unit for electronic skin brachytherapy. J Contemp Brachytherapy 2014; 6: 45-53.

11. Candela-Juan C, Niatsetski Y, Ouhib Z et al. Commissioning and periodic tests of the Esteya ${ }^{\circledR}$ electronic brachytherapy system. J Contemp Brachytherapy 2015; 7: 189-195.

12. AJCC (American Joint Committee on Cancer). Cancer staging of cutaneous nonmelanoma skin cancer. 2010.

13. Ballester Sánchez R, Pons Llanas O, Pérez Calatayud J et al. Dermoscopy margin delineation in radiotherapy planning for superficial or nodular basal cell carcinoma. Br J Dermatol 2014; 172: 1162-1163.

14. Ballester-Sánchez R, Pons-Llanas O, Llavador-Ros $\mathrm{M}$ et al. Depth determination of skin cancers treated with superficial brachytherapy: ultrasound vs histopathology. J Contemp Brachytherapy 2014; 6: 356-361.

15. Tormo A, Celada F, Rodriguez S et al. Non-melanoma skin cancer treated with HDR Valencia applicator: clinical outcomes. J Contemp Brachytherapy 2014; 6: 167-172.

16. Cho M, Gordon L, Rembielak A, Woo TC. Utility of radiotherapy for treatment of basal cell carcinoma: a review. Br J Dermatol 2014; 171: 968-973.

17. Hall VL, Leppard BJ, McGill J et al. Treatment of basal-cell carcinoma: comparison of radiotherapy and cryotherapy. Clin Radiol 1986; 37: 33-34.

18. Avril MF, Auperin A, Margulis A et al. Basal cell carcinoma of the face: surgery or radiotherapy? Results of a randomized study. Br J Cancer 1997; 76: 100-106.

19. Nguyen NP, Ries T, Vock J et al. Effectiveness of radiotherapy for elderly patients with nonmelanoma skin cancer of the head. Geriatr Gerontol Int 2015; 15: 601-605.

20. Bhatnagar A. Nonmelanoma skin cancer treated with electronic brachytherapy: results at 1 year. Brachytherapy 2013; 12: 134-140.

21. Khan L, Choo R, Breen D et al. Recommendations for CTV margins in radiotherapy planning for non-melanoma skin cancer. Radiother Oncol 2012; 104: 263-266.

22. Carducci M, Bozzetti M, De Marco G et al. Usefulness of margin detection by digital dermoscopy in the traditional sur- 
gical excision of basal cell carcinomas of the head and neck including infiltrative/morpheaform type. J Dermatol 2012; 39: 326-330.

23. Carducci M, Bozzetti M, Foscolo AM et al. Margin detection using digital dermatoscopy improves the performance of traditional surgical excision of basal cell carcinomas of the head and neck. Dermatol Surg 2011; 37: 280-285.

24. Nassiri-Kashani M, Sadr B, Fanian F et al. Pre-operative assessment of basal cell carcinoma dimensions using high frequency ultra-sound and its correlation with histopathology. Skin Res Technol 2013; 19: 132-138.

25. Hernández C, del Boz J, de Troya M. Can high-frequency skin ultrasound be used for the diagnosis and management of basal cell carcinoma? Actas Dermosifiliogr 2014; 105: 107-111. 